Research Article

\title{
FIR to FIR Model Reduction with Linear Group Delay in Passband by SDP Optimization
}

\author{
Haijiang Hu, ${ }^{1}$ Shaojing Song $\mathbb{D}^{1}{ }^{1}$ and Fengdeng Zhang ${ }^{2}$ \\ ${ }^{1}$ College of Engineering, Shanghai Polytechnic University, 201209 Shanghai, China \\ ${ }^{2}$ School of Optical-Electrical and Computer Engineering, University of Shanghai for Science and Technology, \\ 200093 Shanghai, China \\ Correspondence should be addressed to Shaojing Song; sjsong@sspu.edu.cn
}

Received 22 September 2019; Revised 15 December 2019; Accepted 9 January 2020; Published 20 February 2020

Academic Editor: Panajotis Agathoklis

Copyright (C) 2020 Haijiang $\mathrm{Hu}$ et al. This is an open access article distributed under the Creative Commons Attribution License, which permits unrestricted use, distribution, and reproduction in any medium, provided the original work is properly cited.

\begin{abstract}
Filter model reduction is an important optimization method in digital signal processing. A method of FIR to FIR model reduction using SDP optimization is proposed in this paper. At first, we use SDP to design an original FIR filter. Then we name a general $K$ order FIR digital filter $H_{1}\left(z^{-1}\right)$ with coefficient values equal to the first $K+1$ filter coefficient values of $H_{0}\left(z^{-1}\right)$. Finally, we design a new general $K$-order FIR digital filter $H_{2}\left(z^{-1}\right)$ connected in parallel with $H_{1}\left(z^{-1}\right)$ using SDP optimization. The experiment results show this method has good performance on the magnitude error and the linear phase in passband. Therefore, this method can be used in the field of digital signal processing.
\end{abstract}

\section{Introduction}

Filter model reduction is an important optimization method in digital signal processing. An FIR filter with the high order has better performance than another FIR filter with the low order. However, when the order is higher, the design of FIR filter is more complex while the implementation of FIR filter is more difficult. Therefore, when the performance requirement is not very rigorous, we use the method of FIR model reduction to design an FIR filter with the lower order instead of another FIR filter with the higher order. In the process of filter design, the convex programming $(\mathrm{CP})$ have already been a hot research for recent years, such as semidefinite programming (SDP) and second-order cone programming (SOCP) [1]. In this paper, we present a method of FIR to FIR model reduction using SDP optimization. The experiment results show this method has good performance on the magnitude error and the linear phase in passband. Therefore, this method can be used in the field of digital signal processing.

Many scientists study model reduction filter design and propose many different methods in recent years. An important recent result is that the filter minimization problem - given a filter, find the smallest equivalent filter-is NP-hard [2]. Reference [3] propose a comparative study based on combined special projections and important frequency-weighted model order reduction algorithms to compute optimal approximants for full-order digital filter. In Reference [4], two different algorithms for approximating FIR by IIR filters are treated: truncation of the balanced model and the Hankel-norm optimal approximation. Cain et al. [5] extends the use of the balanced model truncation and Hankel-norm optimal approximation to permit close approximation of complex FIR prototypes by IIR filters. Saberifar et al. [6] considers automatic reduction of combinatorial filters to a given size, even if that reduction necessitates changes to the filter's behavior. Agrawal et al. [7] proposes an efficient technique for optimal design of IIR filter with minimum passband error, minimum stopband error, high stopband attenuation, and also free from limit cycle effect using cuckoo search algorithm. Yu and Xiong [8] studies an $H \infty$ model reduction problem for interval frequency negative imaginary systems. Ramesh et al. [9] propose that the higher-order discrete systems are 
approximated into the lower-order discrete systems with the help of proposed model order reduction technique. This order reduction includes cross multiplication of polynomials process and using the Genetic Algorithm at the tail end of approximation process. Cain et al. [5] extends the use of the balanced model truncation and Hankel-norm optimal approximation to permit close approximation of complex FIR prototypes by IIR filters. Aldhaheri [10] presents a numerically efficient technique for the design of reduced-order IIR digital filters. Deng et al. [11] studies the design of linear phase IIR filters via optimal Hankel-norm approximation. Adamou-Mitiche and Mitiche [12] uses the special projections of frequency-weighted model order reduction algorithms, based on the gradient flow without frequency weighted, the frequency-weighted balanced truncation, and the gradient flow with frequency weighted, respectively. Reference [13] proposes a new Hankel-norm approach to the robust receding horizon FIR filter design in discrete-time state space under intensive external disturbances. In contrast to the above methods, our proposed method has simple process and good performance. Therefore, this method is easy to apply in the model reduction filter design.

\section{Principle}

In our design, we use the following method to realize the FIR to FIR model reduction.
2.1. Basic SDP Optimization for FIR Design. In our design, $H_{0}(\omega)$ is a FIR filter with $N$-order. $H_{0}(\omega)$ is stated as follows:

$$
H_{0}\left(e^{-j \omega}\right)=\sum_{n=0}^{N} h_{n} e^{-j n \omega},
$$

where $N$ is the order of filter.

$H_{0}(\omega)$ is also stated as follows:

$$
\begin{aligned}
H_{0}(\omega) & =\sum_{n=0}^{N} h_{n} e^{-j n \omega} \\
& =h^{T}(c(\omega)-j s(\omega)) .
\end{aligned}
$$

We define $F(x)=\operatorname{diag}\left\{D\left(\omega_{1}\right), D\left(\omega_{2}\right), \ldots, D\left(\omega_{M}\right)\right\}$, where $M$ is the number of sampling points, and

$$
D(\omega)=\left[\begin{array}{ccc}
\delta & \alpha_{1}(\omega) & \alpha_{2}(\omega) \\
\alpha_{1}(\omega) & 1 & 0 \\
\alpha_{2}(\omega) & 0 & 1
\end{array}\right] \geq 0 .
$$

In $D(\omega)$,

$$
\begin{aligned}
& \alpha_{1}(\omega)=h^{T} W(\omega) c(\omega)-W(\omega) H_{r}(\omega), \\
& \alpha_{2}(\omega)=h^{T} W(\omega) s(\omega)-W(\omega) H_{i}(\omega),
\end{aligned}
$$

where $W(\omega)$ is the weight function, $H_{r}(\omega)$ and $H_{i}(\omega)$ are the real part and the imaginary part of transfer function in the ideal filter. And we can obtain

$$
\begin{aligned}
D(\omega)= & {\left[\begin{array}{lll}
1 & 0 & 0 \\
0 & 0 & 0 \\
0 & 0 & 0
\end{array}\right] \delta+\left[\begin{array}{ccc}
0 & W(\omega) \cos (0 \omega) & W(\omega) \sin (0 \omega) \\
W(\omega) \cos (0 \omega) & 0 & 0 \\
W(\omega) \sin (0 \omega) & 0 & 0
\end{array}\right] h_{0} } \\
& +\cdots+\left[\begin{array}{cccc}
0 & W(\omega) \cos (N \omega) & W(\omega) \sin (N \omega) \\
W(\omega) \cos (N \omega) & 0 & 0 \\
W(\omega) \sin (N \omega) & 0 & 0
\end{array}\right] h_{N}+\left[\begin{array}{ccc}
0 & -W(\omega) H_{r}(\omega) & W(\omega) H_{i}(\omega) \\
-W(\omega) H_{r}(\omega) & 1 \\
W(\omega) H_{i}(\omega) & 0
\end{array}\right],
\end{aligned}
$$

and

$D(\omega)=D_{1}(\omega) \delta+D_{2}(\omega) h_{0}+\cdots+D_{N+2}(\omega) h_{N}+D_{0}(\omega)$.

Therefore, for SDP optimization,

$$
\begin{aligned}
& F_{0}=\left[\begin{array}{ccc}
D_{0}\left(\omega_{1}\right) & \cdots & 0 \\
\vdots & \ddots & \vdots \\
0 & \cdots & D_{0}\left(\omega_{M}\right)
\end{array}\right] \\
& F_{1}=\left[\begin{array}{ccc}
D_{1}\left(\omega_{1}\right) & \cdots & 0 \\
\vdots & \ddots & \vdots \\
0 & \cdots & D_{1}\left(\omega_{M}\right)
\end{array}\right] \text {, } \\
& \text { : } \\
& F_{N+2}=\left[\begin{array}{ccc}
D_{N+2}\left(\omega_{1}\right) & \cdots & 0 \\
\vdots & \ddots & \vdots \\
0 & \cdots & D_{N+2}\left(\omega_{M}\right)
\end{array}\right] \text {, }
\end{aligned}
$$


where $M$ is the number of sampling points.

We also can define $F F$ and $c$ as follows: $F F=\left[\begin{array}{llll}F_{1} & F_{2} & \cdots & F_{N+2}\end{array}\right]$ and $c=\left[\begin{array}{llll}1 & 0 & \cdots & 0\end{array}\right]^{T}$. epsi is an presetting threshold value and a constant. After setting $F_{0}$, $F F, c$, and epsi, we can get the result from output $x s$ by SDP method. After all parameters are obtained, we use SeDuMi [14] for Matlab to optimize this problem. The SDP function in SeDuMi is defined as follows:

function $\left[x s, F_{s}, f_{s}, k\right]=$ projective_sdp $\left(c, F F, F_{0}\right.$, epsi $)$

Title: projective algorithm for homogenized SDP problems.

Description: implements the projective algorithm.

It finds a vector $x$ that

minimizes $c^{\prime} * x$

subject to: $F(x) \geq 0$

where $\quad F(x)=x(1) * F_{1}+x(2) *$

$F_{2}+\cdots+x(n) * F_{n}+F_{0}$ is positive semidefinite.

Input:

$c: p$-dimensional cost vector

FF: $\quad m \times m * p$ matrix of the form $F F=\left[\begin{array}{llll}F_{1} & F_{2} & \cdots & F_{p}\end{array}\right]$

$F_{0}: m \times m$ constant matrix constraint $F(x) \geq 0$

epsi: tolerance for the gap between $f(x k)$ and its lower bound

Output:

$x s$ : solution for the decision variables

$f_{s}$ : value of the objective function at $x=x s$

$F_{s}$ : constraint matrix $F(x)$ at $x=x s$

$k$ : number of iterations at convergence
2.2. FIR Model Reduction by SDP. At first, we name a general $K$-order FIR digital filter $H_{1}\left(z^{-1}\right)$ with coefficient values equal to the first $K+1$ filter coefficient values of $H_{0}\left(z^{-1}\right)$. $K$ is a positive integer, which is not less than $N / 2$. Then, we design a new general $K$-order FIR digital filter $H_{2}\left(z^{-1}\right)$ connected in parallel with $H_{1}\left(z^{-1}\right)$ using SDP optimization.

This step can be described that the objective is to design $H_{2}\left(z^{-1}\right)$ to minimize both the magnitude difference and group delay difference between $H_{1}\left(z^{-1}\right)+H_{2}\left(z^{-1}\right)$ and $H_{0}\left(z^{-1}\right)$. Therefore, the objective function is stated as follows:

$$
H_{\mathrm{obj}}(\omega)=\left(h_{1}+h_{2}\right)^{T}(c(\omega)-j s(\omega))
$$

where $h_{1}$ is constant vector and $h_{2}$ is optimizing objective.

$F(x)=\operatorname{diag}\left\{D\left(\omega_{1}\right), D\left(\omega_{2}\right), \ldots, D\left(\omega_{M}\right)\right\}$, where $M$ is number of sampling points, and

$$
D(\omega)=\left[\begin{array}{ccc}
\delta & \alpha_{1}(\omega) & \alpha_{2}(\omega) \\
\alpha_{1}(\omega) & 1 & 0 \\
\alpha_{2}(\omega) & 0 & 1
\end{array}\right] \geq 0 .
$$

In $D(\omega)$,

$$
\begin{aligned}
& \alpha_{1}(\omega)=\left(h_{1}+h_{2}\right)^{T} W(\omega) c(\omega)-W(\omega) H_{r}(\omega), \\
& \alpha_{2}(\omega)=\left(h_{1}+h_{2}\right)^{T} W(\omega) s(\omega)-W(\omega) H_{i}(\omega),
\end{aligned}
$$

where $W(\omega)$ is the weight function and $H_{r}(\omega)$ and $H_{i}(\omega)$ are the real part and the imaginary part of transfer function in the ideal filter. And we can obtain

$$
\begin{aligned}
& D(\omega)=\left[\begin{array}{lll}
1 & 0 & 0 \\
0 & 0 & 0 \\
0 & 0 & 0
\end{array}\right] \delta+\left[\begin{array}{ccc}
0 & W(\omega) \cos (0 \omega) & W(\omega) \sin (0 \omega) \\
W(\omega) \cos (0 \omega) & 0 & 0 \\
W(\omega) \sin (0 \omega) & 0 & 0
\end{array}\right] h_{10}+\cdots \\
& +\left[\begin{array}{ccc}
0 & W(\omega) \cos (K \omega) & W(\omega) \sin (K \omega) \\
W(\omega) \cos (K \omega) & 0 & 0 \\
W(\omega) \sin (K \omega) & 0 & 0
\end{array}\right] h_{1 K}+\left[\begin{array}{ccc}
0 & W(\omega) \cos (0 \omega) & W(\omega) \sin (0 \omega) \\
W(\omega) \cos (0 \omega) & 0 & 0 \\
W(\omega) \sin (0 \omega) & 0 & 0
\end{array}\right] h_{20} \\
& +\cdots+\left[\begin{array}{ccc}
0 & W(\omega) \cos (K \omega) & W(\omega) \sin (K \omega) \\
W(\omega) \cos (K \omega) & 0 & 0 \\
W(\omega) \sin (K \omega) & 0 & 0
\end{array}\right] h_{2 K}+\left[\begin{array}{ccc}
0 & -W(\omega) H_{r}(\omega) & W(\omega) H_{i}(\omega) \\
-W(\omega) H_{r}(\omega) & 1 & 0 \\
W(\omega) H_{i}(\omega) & 0 & 1
\end{array}\right] \text {. }
\end{aligned}
$$

and

$$
D(\omega)=D_{1}(\omega) \delta+D_{2}(\omega) h_{0}+\cdots+D_{K+2}(\omega) h_{K}+D_{0}(\omega)
$$

The following analysis is same to basic SDP optimization. After obtaining $H_{2}\left(z^{-1}\right)$, we calculate $H_{1}\left(z^{-1}\right)+H_{2}\left(z^{-1}\right)$ as the new FIR filter, and compare the performance between $H_{0}\left(z^{-1}\right)$ and $H_{1}\left(z^{-1}\right)+H_{2}\left(z^{-1}\right)$. 

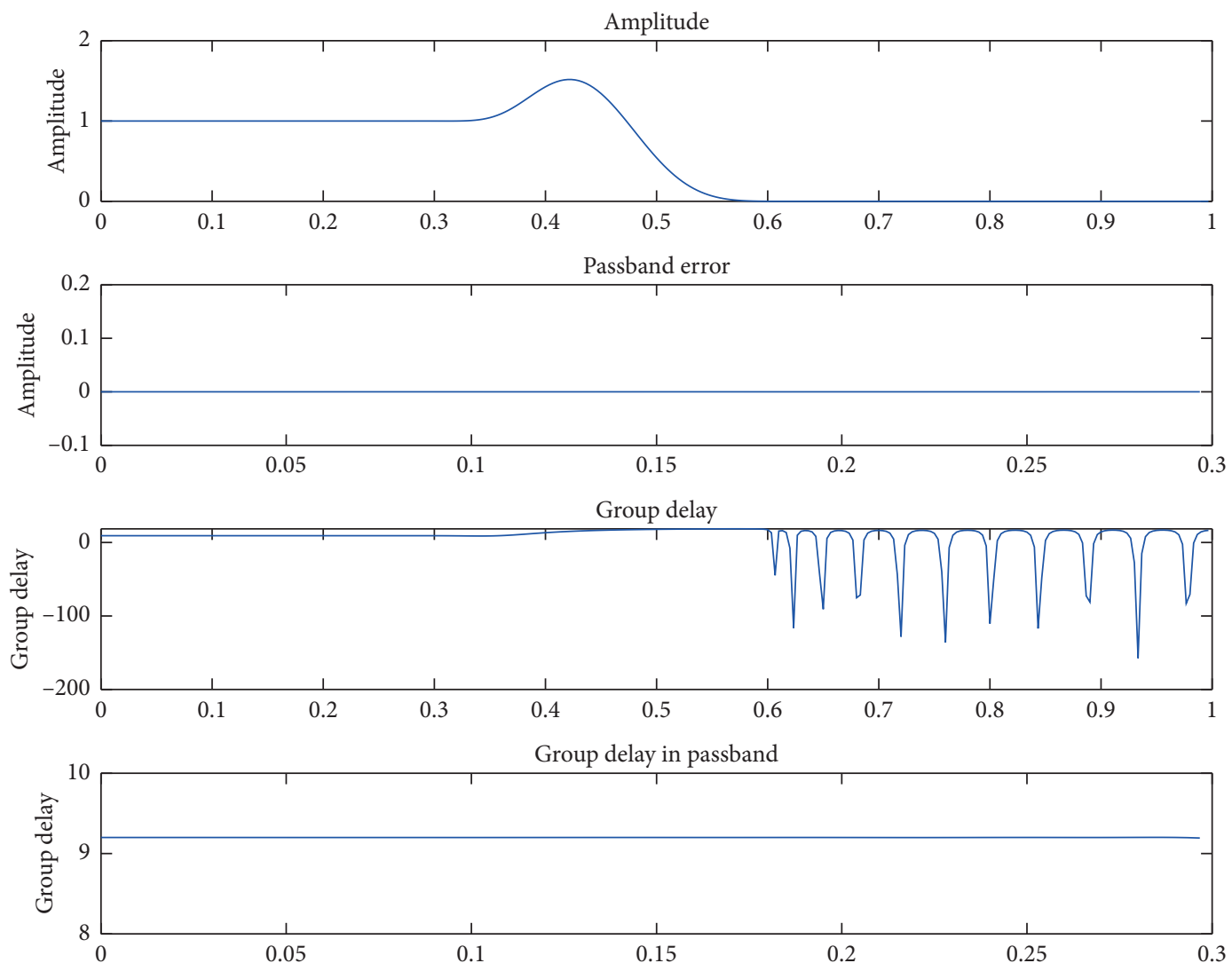

FIgure 1: Performance of original FIR filter for example 1.
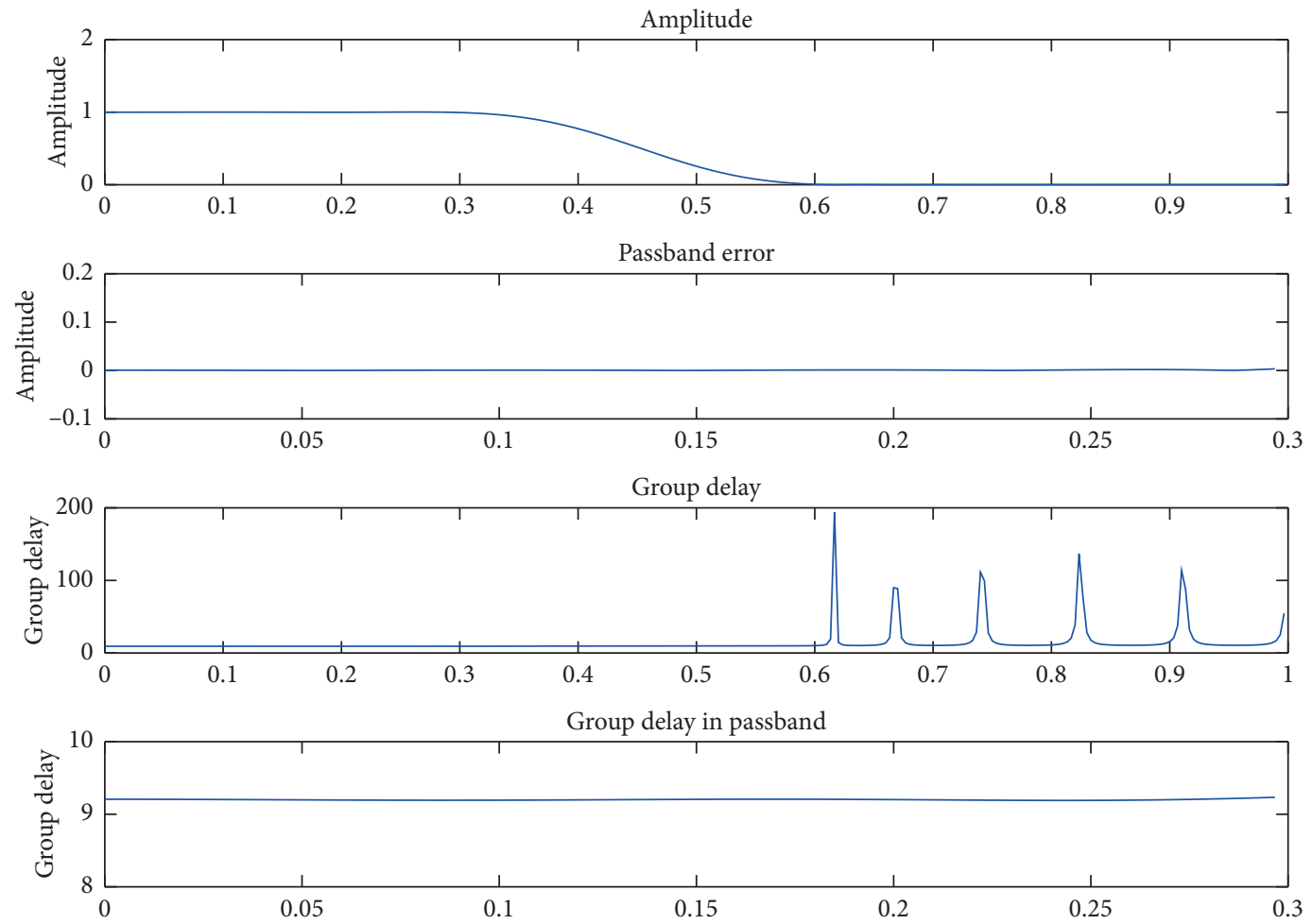

FIgURe 2: Performance of model reduction FIR filter for example 1. 
TABle 1: Performance comparison in example 1.

\begin{tabular}{lccc}
\hline Design method & \multicolumn{1}{c}{ Performance } & $\delta_{q}$ \\
\hline$H_{0}$ & 0.00000517 & 0.00002981 & 0.00653796 \\
$H_{1}$ & 0.09134123 & 0.16381954 & 1.19473802 \\
$H_{1}+H_{2}$ & 0.00340258 & 0.00634723 & 0.03373886 \\
\hline
\end{tabular}
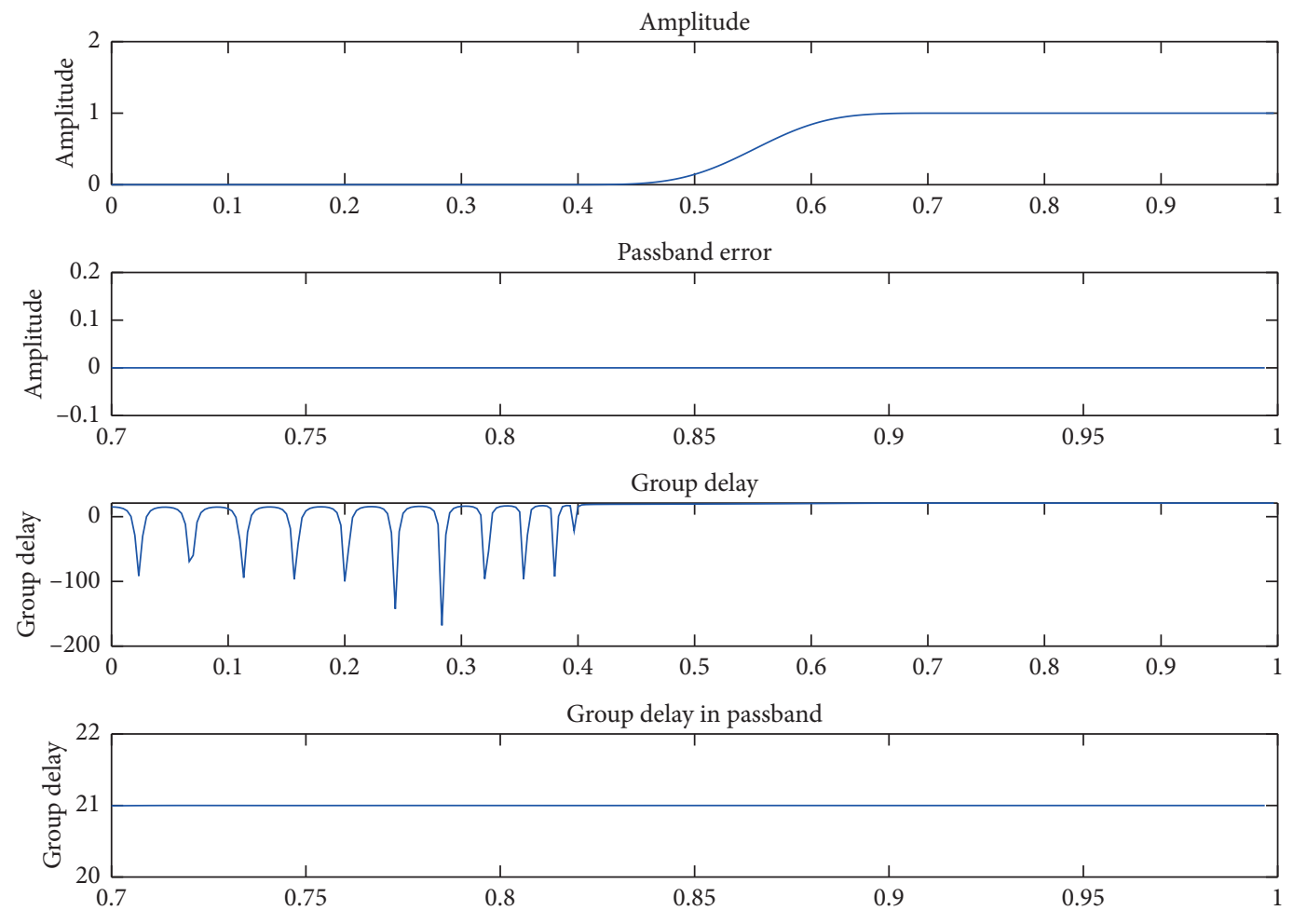

FIgURe 3: Performance of original FIR filter for example 2.

\section{Experiments}

In the following two examples, we use our method to implement the model reduction FIR design. In our design, the number of sampling point $M$ is 900 , epsi is 0.1 , and weight function $W(\omega)$ is

$$
W(\omega)= \begin{cases}1, & \omega \text { in passband, } \\ 1.5, & \omega \text { in stopband, } \\ 0, & \omega \text { in transition band, }\end{cases}
$$

respectively. The first example is to design the low-pass model reduction FIR filter and the second example is to design the high-pass model reduction FIR filter.

3.1. Example 1. For the low pass filter example: original filter order $=40$, model reduction filter order $=20, w_{p}=0.3 \pi$, $w_{s}=0.6 \pi$, passband group delay $=9.2$. This FIR filter is described as follows:

$$
H_{\text {ideal }}(\omega)= \begin{cases}e^{-j 9.2 \omega}, & \omega \leq 0.3 \pi \\ 0, & \omega>0.6 \pi\end{cases}
$$

The design results of the second experiment are shown in Figures 1 and 2. Figure 1 shows the result of original FIR filter with 40 -order while Figure 2 shows the result of FIR filter with 20-order after model reduction. From Figures 1 and 2, we also can know the magnitude-frequency characteristic of model reduction FIR filter is similar to original FIR filter, while the group delay in passband of model reduction FIR filter is also the approximate linearization. Table 1 shows the passband error $\delta_{p}$, the stopband error $\delta_{s}$, and the group delay error $\delta_{q}$ of the original FIR filter and the model reduction FIR filter in experiment 1 . From Table 1, we can see the performance on the passband error, the stopband error, and the group delay in passband in the model reduction FIR filter are similar to the performance of the original FIR filter. Therefore, our method is useful for FIR to FIR model reduction with linear group delay in passband.

3.2. Example 2. For a high pass filter example: original filter order $=40$, model reduction filter order $=30, w_{s}=$ $0.4 \pi, w_{p}=0.7 \pi$, passband group delay $=21$. This FIR filter is described as follows: 

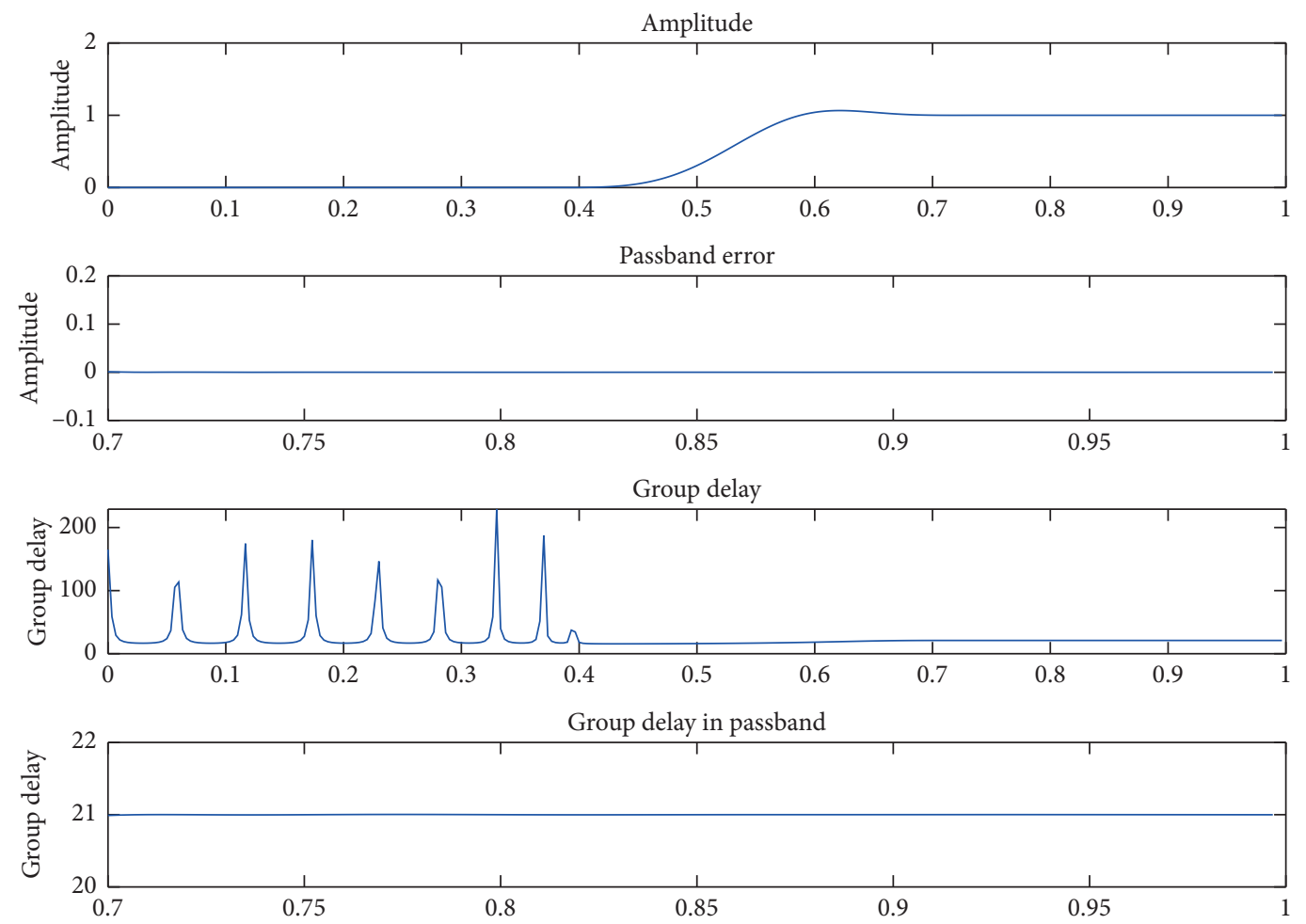

FIGURe 4: Performance of model reduction FIR filter for example 2.

TABle 2: Performance comparison in example 2.

\begin{tabular}{lccc}
\hline Design method & $\delta_{p}$ & $\delta_{s}$ & $\delta_{g}$ \\
\hline$H_{0}$ & 0.00007143 & 0.00001533 & 0.00458099 \\
$H_{1}$ & 0.00788919 & 0.00690130 & 0.07617960 \\
$H_{1}+H_{2}$ & 0.00106166 & 0.00027860 & 0.01039840 \\
\hline
\end{tabular}

$$
H_{\text {ideal }}(\omega)= \begin{cases}0, & \omega \leq 0.4 \pi, \\ e^{-j 21 \omega}, & \omega>0.7 \pi .\end{cases}
$$

The design results of the third experiment are shown in Figures 3 and 4 . Figure 3 shows the result of original FIR filter with 40 -order while Figure 4 shows the result of FIR filter with 30-order after model reduction. From Figures 3 and 4, we also can know the magnitude-frequency characteristic of model reduction FIR filter is similar to original FIR filter, while the group delay in passband of model reduction FIR filter is also the approximate linearization. Table 2 shows the passband error $\delta_{p}$, the stopband error $\delta_{s}$, and the group delay error $\delta_{g}$ of the original FIR filter and the model reduction FIR filter in experiment 2. From Table 2, we can see the performance on the passband error, the stopband error, and the group delay in passband in the model reduction FIR filter are similar to the performance of the original FIR filter. Therefore, in high pass FIR filter design, our method is also useful for FIR to FIR model reduction with linear group delay in passband.

\section{Conclusion}

In this paper, we use SDP to solve FIR to FIR model reduction design. At first, we name a general $K$-order FIR digital filter $H_{1}\left(z^{-1}\right)$ with coefficient values equal to the first $K+1$ filter coefficient values of $H_{0}\left(z^{-1}\right)$. Then we design a new general $K$-order FIR digital filter $H_{2}\left(z^{-1}\right)$ connected in parallel with $H_{1}\left(z^{-1}\right)$ using SDP optimization. The experiments testify this method has good performance on the passband error and group delay error in the passband. Therefore, this method is an efficient algorithm for FIR to FIR model reduction which can be used in the digital signal processing widely.

\section{Data Availability}

The data used to support the findings of this study are available from the corresponding author upon request.

\section{Conflicts of Interest}

The authors declare that they have no conflicts of interest.

\section{Acknowledgments}

The generous support of the Discipline Construction Fund of SSPU (XXKZD1605) and the Construction of University Enterprise Cooperation Automobile Electronic Joint 
Experiment Center (A11NH182016) are gratefully acknowledged.

\section{Supplementary Materials}

This file shows the experiment data in our manuscript, which include a low-pass FIR design and a high-pass FIR design. (Supplementary Materials)

\section{References}

[1] G. Beylkin, R. D. Lewis, and L. Monzon, "On the design of highly accurate and efficient IIR and FIR filters," IEEE Transactions on Signal Processing, vol. 60, no. 8, pp. 40454054, 2012.

[2] F. Z. Saberifar, A. Mohades, M. Razzazi, and J. M O'Kane, "Combinatorial filter reduction: special cases, approximation, and fixed-parameter tractability," Journal of Computer and System Sciences, vol. 85, pp. 74-92, 2017.

[3] A. B. H. Adamou-Mitiche and L. Mitiche, "Internal projections and $L_{2}$ norm for optimal digital filters model reduction: a comparative study," Journal of Circuits, Systems and Computers, vol. 28, no. 1, Article ID 1930001, 2019.

[4] I. Kale, J. Gryka, G. D. Cain et al., "FIR filter order reduction: balanced model truncation and Hankel-norm optimal approximation," IEE Proceedings-Vision, Image and Signal Processing, vol. 141, no. 3, pp. 168-174, 1994.

[5] G. D. Cain, J. Gryka, and I. Kale, "Complex IIR filter design through balanced model reduction of FIR prototypes," Electronics Letters, vol. 31, no. 16, pp. 1332-1334, 1995.

[6] F. Z. Saberifar, A. Mohades, M. Razzazi, and J. M. O'Kane, "Improper filter reduction," Journal of Algorithms and Computation, vol. 50, no. 1, pp. 1-35, 2017.

[7] N. Agrawal, A. Kumar, V. Bajaj, and G. K. Singh, "High order stable infinite impulse response filter design using cuckoo search algorithm," International Journal of Automation and Computing, vol. 14, no. 5, pp. 91-104, 2017.

[8] L. Yu and J. Xiong, "Ho model reduction for interval frequency negative imaginary systems," IEEE Transactions on Circuits and Systems I, vol. 66, no. 3, pp. 1116-1129, 2019.

[9] K. Ramesh, R. G. Ganesan, and K. Mahalakshmi, "Approximation and optimization of discrete systems using order reduction technique," Energy Procedia, vol. 117, pp. 761-768, 2017.

[10] R. W. Aldhaheri, "Frequency-domain model reduction approach to design IIR digital filters using orthonormal bases," AEU-International Journal of Electronics and Communications, vol. 60, no. 6, pp. 413-420, 2006.

[11] N. Deng, S. Shao, and G. Gu, "Design of linear phase IIR filters via optimal Hankel-norm approximation," Control Theory \& Applications, vol. 24, no. 2, pp. 200-204, 2007.

[12] A. B. H. Adamou-Mitiche and L. Mitiche, "Digital filters approximation based on optimal weighted approaches: a comparative study," in Proceedings of the 4th International Conference on Control Engineering \& Information Technology, December 2016.

[13] A. C. Ki, S. Yuriy, and Z. Shunyi, "Hankel-norm approach to robust FIR estimation of dynamic systems under external disturbances," IEEE/ASME Transactions on Mechatronics, vol. 23, no. 4, pp. 1973-1980, 2018.

[14] http://sedumi.ie.lehigh.edu. 